\title{
CLEM takes a polar plunge
}

\author{
Combining cryogenic super-resolution microscopy and focused ion beam scanning electron microscopy allows \\ nanoscale views of protein architecture in the context of cellular ultrastructure in whole cells.
}

$\mathrm{C}$ orrelative light and electron microscopy (CLEM) combines the benefits of fluorescence microscopy for imaging specifically labeled cellular components with the high-resolution ultrastructure imaging provided by electron microscopy, and has yielded numerous insights into biological processes and subcellular anatomy. Inspired by technical advances in both super-resolution fluorescence microscopy and scanning electron microscopy, David Hoffman and Gleb Shtengel, in the laboratories of Eric Betzig and Harald Hess at the Janelia Research Campus of the Howard Hughes Medical Institute, developed an improved correlative imaging workflow that provides high-resolution views of whole cells in three dimensions.

A key to their workflow was carrying out the super-resolution light microscopy under cryogenic conditions, which had a clear impact on both the quality of the fluorescence and subsequent electron microscopy. "Initially we knew that fluorescence at cryogenic temperatures could be highly stable, immortal, and very difficult to bleach," notes Hess. "In other words, structured illumination microscopy, SIM, which can be limited by bleaching at room temperature, should perform better with cryogenic samples. So we set out to make a cryogenic SIM."

To prepare their samples, they first carried out cryofixation with high-pressure freezing before moving the samples to a cryostat for fluorescence imaging. Their cryogenic SIM featured a moving objective to allow it to image samples in the stationary cryostat. The team found that they could readily do rapid three-color imaging with minimal photobleaching with their cryogenic SIM. They used this relatively fast imaging process to screen a large number of cells to identify potentially interesting cells for imaging at even higher resolution with single-molecule localization microscopy. This prescreening step was crucial, as the $3 \mathrm{D}$ localization microscopy took 1-2 days per cell.

These localization microscopy experiments revealed unexpected details of the photophysical behaviors of multiple fluorescent proteins and dyes at cryogenic temperatures and identified many of the

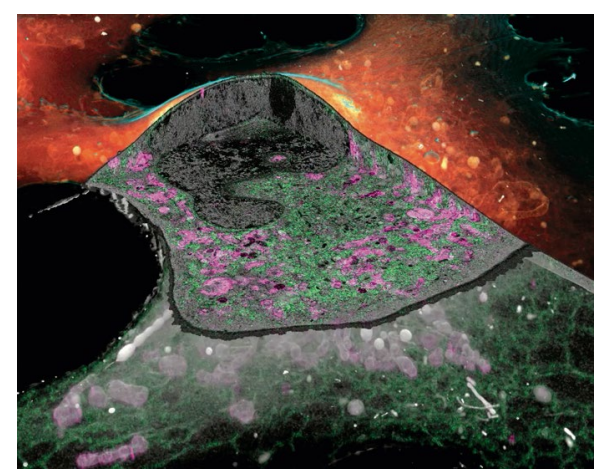

Correlative cryogenic super-resolution imaging and FIB-SEM of a whole cell. Adapted with permission from Hoffman, D. P. et al. Science 367, eaaz5357 (2020), AAAS.

probes as ideal for cryogenic localization microscopy, an observation that surprised Hess. "I never expected some cryogenic fluorescent proteins to have additional strong, high-contrast blinking properties that could be harnessed to make high-quality superresolution images of subcellular details."

Following fluorescence microscopy, the cells were subjected to freeze substitution, resin embedding, and staining to prepare them for imaging by focused ion beam scanning electron microscopy (FIB-SEM). In FIB-SEM, samples are imaged over multiple rounds that consist of SEM imaging followed by FIB milling, which exposes a new layer of the specimen for imaging to build up a composite $3 \mathrm{D}$ image of the sample. The process took the team up to 15 days per cell. The researchers took care to ensure the same cells were imaged by both methods and to register the images from the different modalities in three dimensions.

With the pipeline in place, the team studied the complex structures of cellular organelles including the mitochondria and endoplasmic reticulum (ER), where they immediately made observations that would have been impossible without their correlative approach. For example, they saw clusters of an ER protein in their localization microscopy data that, when registered to the SEM data, localized to varicosities in ER tubules. They note that these observations might otherwise have been dismissed as artifacts. They also observed small vesicles to be ubiquitous throughout the cell, but were able to pinpoint some through specific labeling and location that may be derived from mitochondria. They even observed a few ER-derived vesicles, whose origin is not fully understood, within the nuclei of cells.

They also applied their approach to study the endolysosomal system, which they note is known for being sensitive to fixation and protein overexpression artifacts. Here they observed many structures that were sufficiently convoluted so as not be apparent by FIB-SEM alone. Furthermore, the researchers examined the structure of cellcell adhesions and nuclear arrangements associated with cerebellar granule neuron progenitor differentiation.

Despite their clear successes in implementing their correlative imaging, Hess recalls that "trying to conclude the project was not easy," explaining that "there were so many high-value extensions, enhancements and demonstrations that it took almost five years before we found an appropriate stopping point to summarize and publish the results."

Hess notes that the immediate next steps for this work involve making the workflow available to biologists, which he notes can be facilitated by an incubating facility such as the Advanced Imaging Center at Janelia. He is realistic, though, and says, "A transition to mainstream is not easy, and it takes many iterations to streamline the methodology, explore the extent of its applicability to diverse biological problems, and to educate biologists of such new capability. If it is compelling and a significant market exists, then commercial companies could complete the transition to mainstream." Given the unprecedented quality of information shown in their images, a bright future is apparent for this technology.

\section{Rita Strack}

Published online: 4 March 2020 https://doi.org/10.1038/s41592-020-0776-1

Research paper

Hoffman, D. P. et al. Correlative three-dimensional super-resolution and block-face electron microscopy of whole vitreously frozen cells. Science 367 , eaaz5357 (2020). 